\title{
REG3 $\gamma$-deficient mice have altered mucus distribution and increased mucosal inflammatory responses to the microbiota and enteric pathogens in the ileum
}

\author{
LMP Loonen ${ }^{1,2}$, EH Stolte ${ }^{2}$, MTJ Jaklofsky ${ }^{2}$, M Meijerink $^{2}, \mathrm{~J} \mathrm{Dekker}^{1}$, P van Baarlen ${ }^{2}$ and JM Wells ${ }^{1,2}$
}

REG3 $\gamma$ is considered to have a protective role against infection with Gram-positive bacteria due to its bactericidal activity, but evidence from in vivo studies is lacking. We generated a REG3 $\gamma^{-1-}$ mouse, and investigated the effect of lack of REG3 $\gamma$ on intestinal mucus distribution, spatial compartmentalization of bacteria, and expression of innate immunity genes. Infection studies were also performed with Gram-positive and Gram-negative pathogens to investigate the antimicrobial role of REG3 $\gamma$. REG3 $\gamma^{-1-}$ mice display altered mucus distribution, increased bacterial contact with the epithelium, and elevated inflammatory markers in the ileum without histological evidence of pathology. Infection response pathway genes were differentially expressed in both Listeria monocytogenes and Salmonella enteritidis infected REG $3 \gamma^{-1-}$ and wild-type (wt) mice. Higher amounts of myeloperoxidase and interleukin-22 transcripts were present in the ileal mucosa of REG3 $\gamma^{-1-}$ than wt mice, but translocation to the organs was unaffected. We concluded that REG3 $\gamma$ has a protective role against mucosal infection with pathogenic Listeria and Salmonella in vivo. REG3 $\gamma$ is equally distributed throughout the mucus and its absence results in increased epithelial contact with the microbiota resulting in low-grade inflammation. REG3 $\gamma$ can bind to Gram-negative and Gram-positive bacteria and influence mucus distribution in the ileum, properties which may contribute to mucosal protection.

\section{INTRODUCTION}

The mammalian intestine harbors one of the most rich and densely populated microbial communities on earth and several adaptations of the host mucosa have evolved to maintain this overall beneficial relationship. One of the main mechanisms involves sequestering of bacteria in the luminal compartment, to avoid colonization of the epithelial cell surface and invasion by opportunistic pathogens. This is in part achieved through the secretion of secretory (s)IgA, which protects the epithelium by the immune exclusion of microbes ${ }^{1}$ and also by the continuous production and removal of secreted mucin. In the small intestine, the mucus barrier is fortified by the accumulation of several Paneth and epithelial cell-produced antimicrobial peptides and proteins (AMP). ${ }^{2}$ One key human antimicrobial protein is hepatocarcinoma-intestine-pancreas/pancreatitis- associated protein (HIP/PAP) (REG3 $\alpha$, GeneID 5068) that has a murine homolog designated regenerating islet-derived 3 beta (REG3 $\beta$ ).

Mammalian REG proteins consist of a carbohydrate recognition domain and a $\mathrm{N}$-terminal secretion signal. Some REG3 family members implicated in host defense are expressed predominantly in the small intestine, including mouse REG3 $\beta$ and REG3 $\gamma$ (refs. 3-6) and human HIP/PAP. ${ }^{7,8}$ The main environmental triggers for expression of REG3 proteins are intestinal colonization by bacteria, ${ }^{5}$ infection, ${ }^{9,10}$ or inflammatory stimuli driven by mucosal inflammation and damage $^{4,11}$ implicating broad roles of REG3 proteins in mucosal defense and immune homeostasis.

Recombinant and refolded REG3 $\gamma$ and human HIP/PAP have been reported to be bactericidal to Gram-positive bacteria ${ }^{1}$ TI Food and Nutrition, Wageningen, The Netherlands and ${ }^{2}$ Host-Microbe Interactomics group, Department of Animal Sciences, Wageningen University, Wageningen,
The Netherlands. Correspondence: JM Wells (jerry.wells@wur.nl) 
through binding to peptidoglycan. ${ }^{5}$ The bactericidal activity of HIP/PAP is controversial as other authors report that HIP/PAP from humans and rats is not bactericidal, but can induce bacterial aggregation. ${ }^{12,13}$

Loss of the antimicrobial REG3 $\gamma$ function was given as an explanation for the decreased spatial separation of commensals from the small intestinal epithelium in REG3 $\gamma$ knockout mice. ${ }^{14}$ The only caveat being that this spatial separation was not based on visualization of the mucus but inferred by fluorescent detection of the bacteria and epithelial nuclei. The firm mucus layer can easily become detached from the epithelial surface during the histological procedure, something which is only apparent by direct staining. Therefore, it is important to visualize the mucus layer in order to accurately assess changes in bacterial exclusion and the extent to which bacteria penetrate the mucus. In addition to the study on spatial segregation of the microbiota in REG3 $\gamma^{-1-}$ mice, Vaishnava et al. ${ }^{14}$ showed increased abundance of mucosal-adhered bacteria including segmented filamentous bacteria, but no effect on bacterial composition or quantity in the lumen of the ileum.

The aim of this study was to determine the in vivo contribution of REG3 $\gamma$ to protection of the mouse mucosa from infection with Gram-positive Listeria monocytogenes and Gram-negative Salmonella enteritidis. We first generated and characterized a REG3 $\gamma^{-1-}$ mouse lacking segmented filamentous bacteria, including histological and immunohistochemical investigations of the mucus and spatial compartmentalization of bacteria in the intestine. In addition, we investigated the effects of REG3 $\gamma$ deficiency in uninfected and pathogeninfected mice using microarray gene expression, $\mathrm{qPCR}$ and measurements of bacterial translocation, innate and serum biomarkers.

\section{RESULTS}

REG3 $\gamma$ deficiency increases innate inflammatory markers in the ileal mucosa

In REG3 $\gamma^{-1-}$ mice expression of Cre-recombinase leads to deletion of the REG3 $\gamma$ cDNA cloned between the loxP sites in the targeting vector, thereby bringing the enhanced GFP coding sequence under control of the canonical REG3 $\gamma$ promoter (supplement and Supplementary Figure S1 online). REG3 $\gamma^{-1-}$ mice had a similar appearance to wild-type (wt) mice, showing no developmental abnormalities or signs of disease, and similar body weights. As expected, REG3 $\gamma$ was not expressed in the small intestine of REG3 $\gamma^{-1-}$ mice (Figure 1a). Enhanced GFP was expressed in the small intestine (Figure 1b) and pancreas, but not in the liver, spleen, or lungs of REG3 $\gamma^{-1-}$ mice (data not shown). Intestinal expression of REG3 $\beta$ was not altered in REG3 $\gamma^{-/-}$mice (Figure 1c). Interleukin (IL)-22, an inflammatory response marker, was significantly increased in the ileum of REG3 $\gamma^{-1-}$ mice (Figure 1d). Furthermore, increased amounts of myeloperoxidase (MPO), a quantitative marker of polymorphonuclear granulocytes, were measured in the ileum of uninfected REG3 $\gamma^{-1-}$ mice compared with uninfected wt mice (Figure 1e). To further investigate the immune status, a comprehensive panel of 58 serum biomarkers was measured.
Macrophage inflammatory protein-2 (MIP-2) $(P=0.0562)$, monocyte chemotactic protein-1 (MCP-1) $(P=0.0117)$, and macrophage colony stimulating factor (M-CSF)-1 $(P=0.0465)$, all of which are associated with the chemotaxis and development of neutrophils and monocytes, were upregulated in REG3 $\gamma^{-1-}$ compared with wt mice (Supplementary Table S3 online). Fifteen cytokines measured in the serum of REG3 $\gamma^{-1-}$ and wt mice were either not significantly different or below detection limit. To determine whether REG3 $\gamma$ deficiency had an effect on immune responses in mucosal lymphoid tissue, cytokine production was measured in ex vivo stimulated immune cells from MLNs. There were no significant differences in the production of IL-10, tumor necrosis factor (TNF)- $\alpha$, interferon (IFN)- $\gamma$, IL-4, and IL-6 between REG3 $\gamma^{-1-}$ and wt mice (Supplementary Figure S2). Therefore, it appears that REG $3 \gamma^{-l-}$ mice exhibit an increased innate inflammatory activity in the mucosa without evoking systemic inflammation.

\section{REG3 $\gamma$ affects the ileal mucus distribution and spatial separation of microbiota}

The relatively higher amounts of IL-22 and MPO in the ileum of REG $3 \gamma^{-1-}$ mice could have been caused by increased microbial translocation in the absence of REG3 $\gamma$. This prompted us to investigate the intestinal morphology and search for deviations from wt mucosal morphology and the location of microbiota. Crossmon-stained tissue of wt and REG3 $\gamma^{-1-}$ mice showed indistinguishable villus-crypt architecture with similar crypt and villus lengths (data not shown). Interestingly, PAS/AB (Figure 2a,c) and specific staining of mucin with an anti-MUC2 antibody (Figure $\mathbf{2 b}, \mathbf{d}$ ) showed a different mucus distribution in the ileum (Figure 2), but not in the colon (data not shown). In all wt mice, an intensely stained band of mucus was consistently observed, lying as a "blanket" above the tips of the villi, surrounding the ileal content; however, this band was absent in each of the REG3 $\gamma^{-1-}$ mice. QPCR on ileal tissue showed that this differential mucus distribution was not due to differences in expression of Muc2 (data not shown). Moreover, distribution of goblet cells appears to be the same in wt and REG3 $\gamma^{-1-}$ mice. No other secretory and membranebound mucins were differentially expressed in the intestinal tissues. In the small intestine, immunofluorescence staining of the mucus and bacteria showed that small numbers of bacteria were observed in contact with the epithelium in REG3 $\gamma^{-1-}$ mice, but not in wt mice (Figure 3a,b); no differences were observed in the colon (Figure $\mathbf{3 c}$,d). To investigate whether REG $3 \gamma$ was present only in the crypts or throughout the mucus, we used immunofluorescent staining techniques to visualize REG3 $\gamma$ in Carnoy-fixed sections of the ileum (Figure 4). REG3 $\gamma$ was detected throughout the mucus in wt mice (Figure 4a).

The findings described above show that in ileum but not in colon, lack of REG3 $\gamma$ protein leads to increased contact of microbiota with host with concomitant absence of a specific, darkly stained mucus band near the villi tips. Lack of REG3 $\gamma$ did not appear to lead to changes in intestinal crypt depth and villi lengths. Since lack of REG3 $\gamma$ led to increased contact between ileal microbiota and epithelia, we were interested to see if lack of 


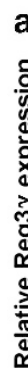

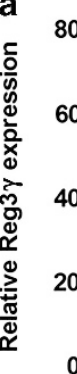
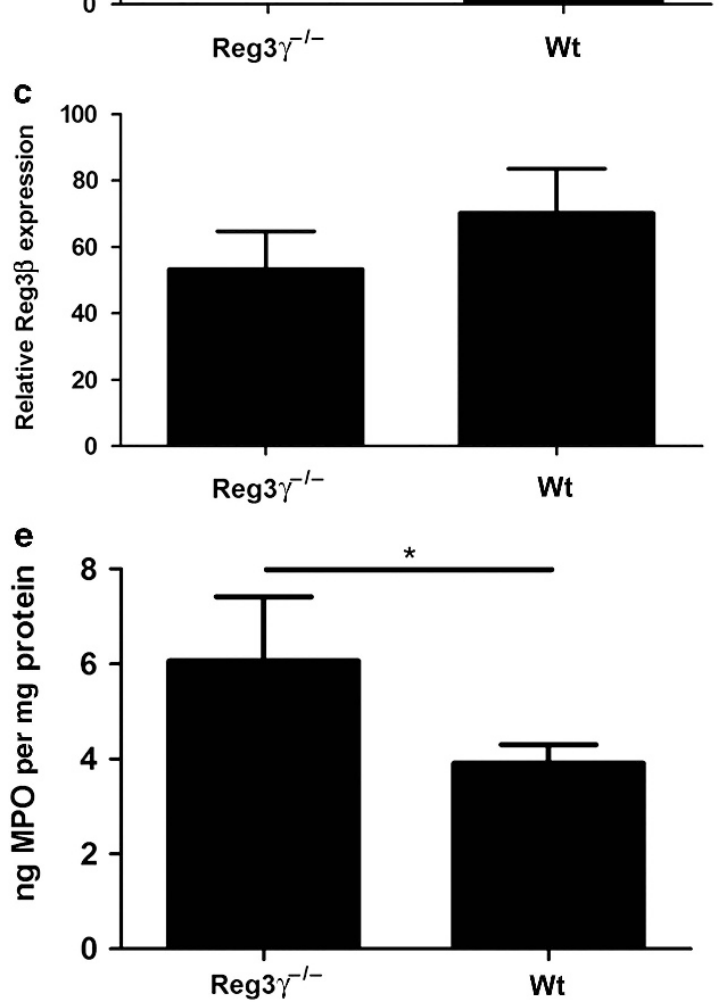
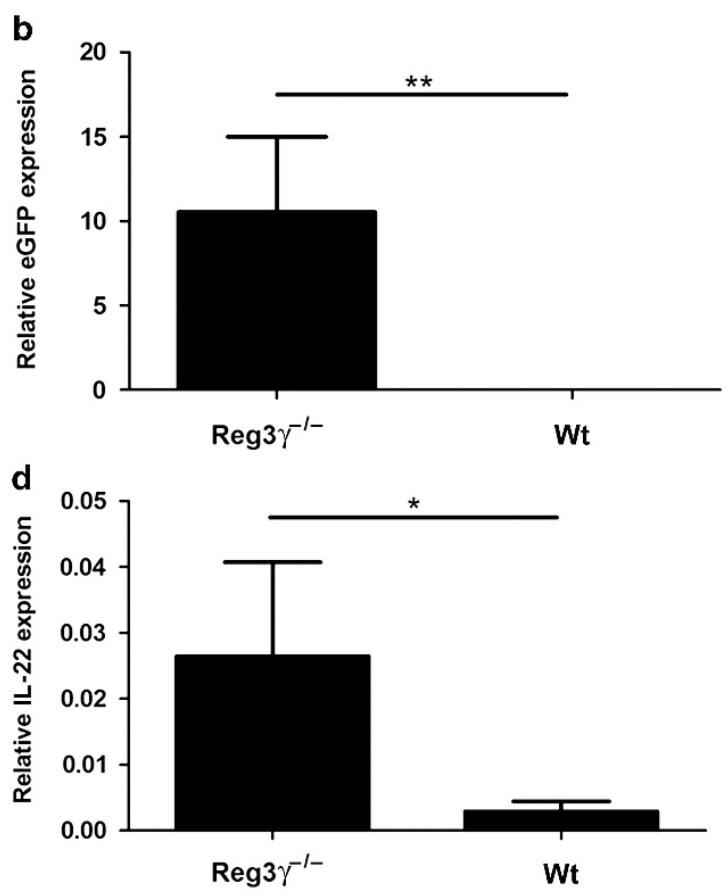

Figure 1 Ileal expression of REG3 $\gamma$, enhanced GFP, REG3 $\beta$, IL-22 and MPO in REG3 $\gamma^{-/-}$and wt mice. Relative mRNA expression determined by qPCR (a-d). ELISA of MPO protein in ileal extracts of control (uninfected) mice used in the infection study $(\mathbf{e}) .{ }^{*}=P<0.05$; ${ }^{* *}=P<0.01$. wt, wild-type.

REG3 $\gamma$ would promote host invasion by an intracellular Grampositive and Gram-negative pathogen.

Mucosal inflammation, but not pathogen translocation to the organs, is increased in infected REG3 $\gamma^{-I-}$ mice

During the course of the infection with $S$. enteritidis and L. monocytogenes, body weight decreased but not significantly compared with non-infected controls (Supplementary Table S4). The colony forming units (CFU) of L. monocytogenes in feces were not significantly different for REG3 $\gamma^{-1-}$ and wt mice. However, significantly higher CFU of S. enteritidis were present in the feces of REG3 $\gamma^{-1-}$ mice compared with wt (Figure 5). No differences were found in translocation of $S$. enteritidis or L. monocytogenes to the spleen, liver, or MLNs (Figure 6a,b). This result was also obtained using a murinized L. monocytogenes strain (Supplementary Figure S3). As a systemic marker of bacterial translocation and inflammation, we measured SAA2 levels in serum. In uninfected mice, the levels of serum SAA2 were below the detection limit of the assay $\left(<7.8 \mathrm{ng} \mathrm{ml}^{-1}\right)$ but levels increased substantially $\left(>10^{5} \mathrm{ng} \mathrm{ml}^{-1}\right)$ after infection with either pathogen. There were no significant differences between SAA2 in REG3 $\gamma^{-1-}$ and wt infected mice (Supplementary Table S5), supporting similar bacterial translocation levels in all infected groups of mice. MPO in ileal tissue was significantly elevated in all infected compared with uninfected mice (Figure 7). Furthermore, in infected mice, MPO levels were increased more in REG3 $\gamma^{-1-}$ than in wt mice.

\section{Gene expression profiles of infected mice}

A transcriptomics approach was used to gain more insight into the potential pathways or mechanisms that might be modulated in the ileum as a consequence of the absence of REG3 $\gamma$ during microbial infection. Infection resulted in the differential $(P<0.02)$ expression of 999 genes by S. enteritidis and 744 genes by L. monocytogenes. Gene ontology (GO) enrichment calculations by ErmineJ and gene set enrichment analysis (GSEA) showed that infection with either pathogen appeared to 

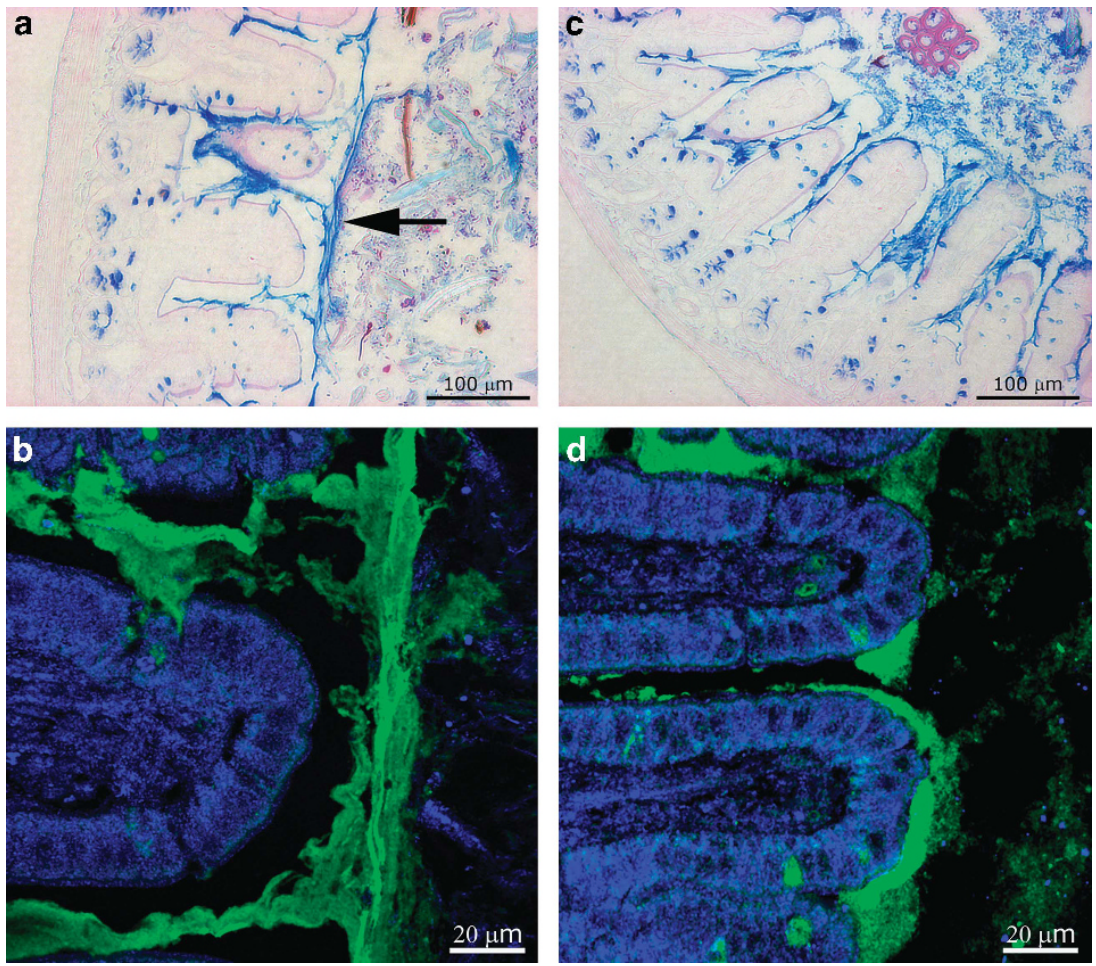

Figure 2 Mucus staining in Carnoy's fixed ileal sections of wt (a, b) and REG3 $\gamma^{-1-}(\mathbf{c}, \mathbf{d})$ mice. PAS/Alcian Blue staining of ileum (a, $\left.\mathbf{c}\right)$. Immunofluorescent detection of MUC2 (b, d) using an anti-MUC2 antibody and goat-anti-rabbit Alexa 488. Arrow in A indicates the continuous layer of mucus overlaying the villi tips. Representative images are shown in each panel. wt, wild-type.

induce mucosal pathways involved in DNA metabolism and cell cycle, IFN signaling and antigen processing and presentation, whereas diverse metabolic pathways including pathways involved in xenobiotic and fatty acid metabolism, PPAR $\alpha$ signaling and peroxisome function appeared to have been suppressed. For detailed network and regulatory pathway analysis, array data were analyzed using Ingenuity Pathway Analysis (IPA).

\section{S. enteritidis induces an IFN-driven immune response via IL-1 $\beta$-MyD88-STAT1/3-IRF axis}

IPA analysis showed that during infection with S. enteritidis, antigen presentation and diverse metabolic and disease pathways were significantly modulated, in addition to pathways involved in immune cell maturation and differentiation. IPA was also used to perform an upstream regulator analysis in order to identify the cascade of upstream transcriptional regulators that could explain the observed gene expression changes in the S. enteritidis infection data set. The regulators that were most strongly induced during infection were If $n-\gamma$, Signal Transducer and Activator of Transcription (Stat)1, IL-1 $\beta$, Stat3, Suppressor of cytokine signaling (Socs)1, Myd88, and Interferon regulatory factor (Irf) 1 suggesting that an IFN-driven immune response via an IL-1 $\beta$-Myd88-Stat1/3-Irf axis was a major determinant of the mouse response to infection. The major cellular responses were involved in activation of hematological system development, immune cell trafficking, cell-cell signaling and interactions, cell fate and an inflammatory response, and a repression of processes involved in infectious disease progression including replication of intracellular pathogens.

\section{L. monocytogenes induces an IFN-driven immune response via TLR3-MyD88-STAT1/3-IRF axis}

IPA analysis showed that during infection with L. monocytogenes, metabolic and antigen presentation pathways and IFN signaling were significantly modulated, in addition to pathways involved in and driven by Janus kinase (JAK)-STAT signaling, the signaling pathway that strongly drives IFN response. Upstream regulator analysis showed that the regulators that were most strongly induced during infection were Stat1, Irf1, Stat3, Toll like receptor (Tlr)3, and Myd88 suggesting that an IFN-driven immune response via a Tlr3-Myd88-Stat1/3-Irf axis was a major determinant of the mouse response to infection. The major cellular responses were involved in (immune) cell fate, an inflammatory response to intracellular pathogens, and a repression of processes involved in small molecule biochemistry, lipid metabolism, and progression of infectious disease. RNA expression of a few infection-related genes was quantified by qPCR, and showed that transcription of Ifn- $\gamma, M i p-2$, and Reg $3 \beta$ were all increased in infected mice compared with uninfected mice (Figure 8a-d). In L. monocytogenes infected mice transcription of Ifn- $\gamma$ was significantly higher in REG3 $\gamma^{-1-}$ than in wt mice (Figure 8d).

\section{In REG3 $\gamma^{-I-}$ mice the infection response is altered compared with wt mice}

To investigate if the transcriptome data revealed relevant differences between infected wt and REG3 $\gamma^{-1-}$ mice, a protein-protein 

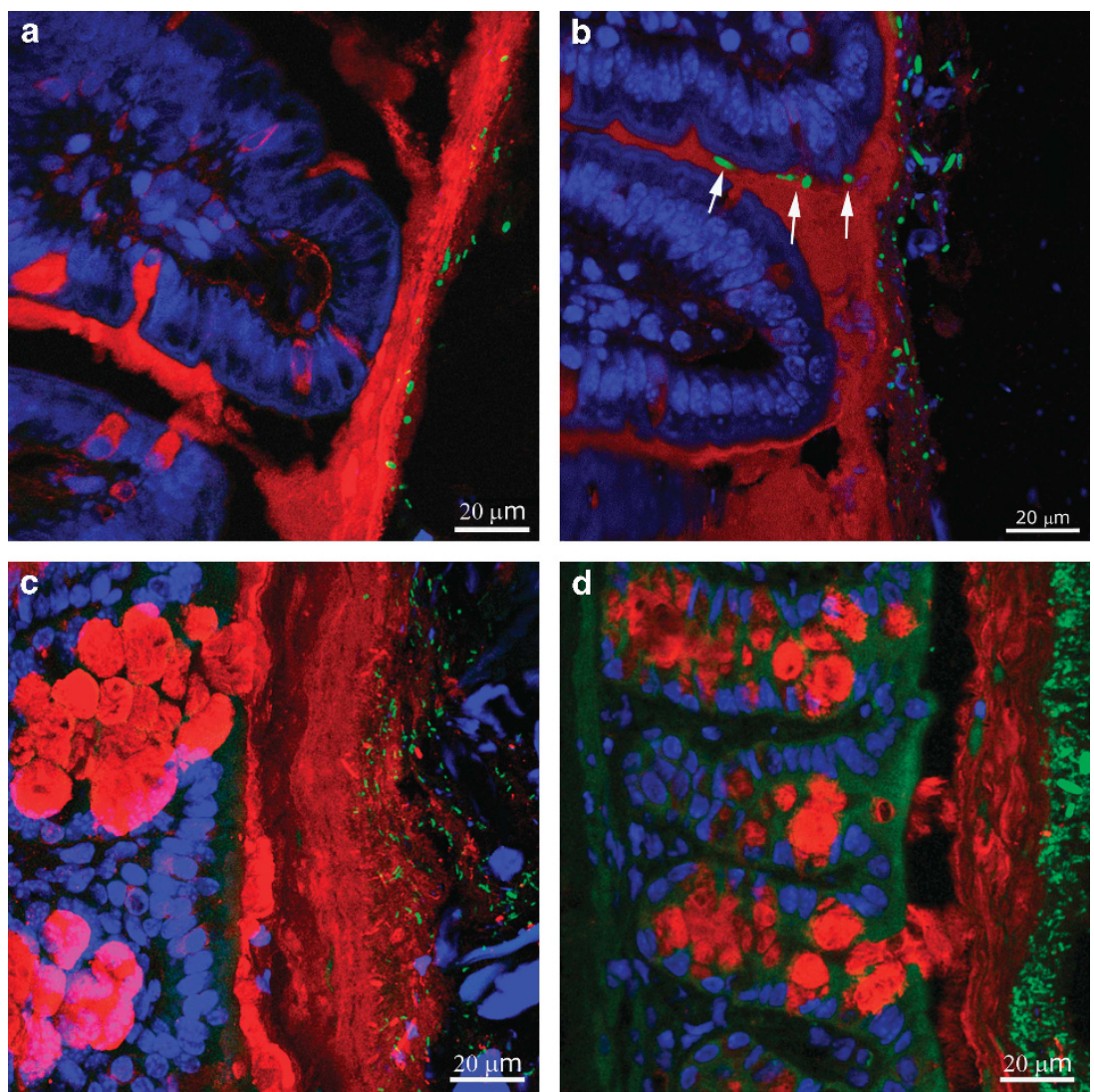

Figure 3 Fluorescent microscopy of mucus and microbiota in Carnoy's fixed sections of ileum and colon from REG3 $\gamma^{-1-}$ and wt mice. Representative images of ileal tissue of wt (a) and REG3 $\gamma^{-1-}$ mice (b); and of colon tissue of wt (c) and REG3 $\gamma^{-1-}$ mice (d). MUC2 was detected by immunofluorescence using anti-MUC2 and goat-anti-rabbit Alexa Cy3 antibodies (red). Nuclei were visualized using DRAQ5 (blue). Bacteria were identified using FISH and the universal Euprobe 338 (green). Arrows in panel b indicate bacteria in contact with the epithelium. wt, wild-type.


Figure 4 REG3 $\gamma$ staining in Carnoy's fixed sections of ileum from wt (a) and REG3 $\gamma^{-1-}$ mice (b), showing distribution of REG3 $\gamma$ throughout the mucus layer in wt animals. A REG3 $\gamma$-specific antibody was detected using a goat-anti-rabbit Alexa 488 secondary antibody. wt, wild-type.

interaction network was generated in IPA for genes with GO terms involved in infectious and bacterial disease. These categories were most significantly enriched in the IPA analyses of mice infected with L. monocytogenes or S. enteritidis. This network was overlaid with gene expression data of infected wt and REG3 $\gamma^{-1-}$ mice. The bacterial disease network included the nodes $M y d 88$, Stat 1/3, Irf1/8, and Socs $1 / 3$ that were regulated both during infection by $S$. enteritidis and by L. monocytogenes.
In S. enteritidis infected mice, the relative expression of 15 of the 80 differentially expressed network genes (19\%) including the genes Ifn- $\gamma, I L-1 \beta$, and Saa was lower in REG3 $\gamma^{-1-}$ mice than in wt mice, whereas $2(3 \%)$ of the network genes (i.e., IFNgamma-induced GTPase and ubiquitin D) were expressed at relatively higher levels in REG3 $\gamma^{-1-}$ compared with wt mice (Supplementary Figure S4). In mice infected with L. monocytogenes, the same comparison showed that 2 of 
the $39(5 \%)$ differentially expressed network genes (Saa and lipocalin 2) were expressed at relatively lower levels during infection of REG3 $\gamma^{-1-}$ mice, whereas 12 genes (31\%) including Ifn- $\gamma, I L-1 \beta, I c a m 1$, and Chemokine (C-X-C motif) ligand $(C x c l) 9 / 10$, were expressed at relatively higher levels (Supplementary Figure S5).

\section{DISCUSSION}

To investigate the role and function of REG3 $\gamma$ in vivo, we generated a REG3 $\gamma^{-1-}$ mouse model that expresses an enhanced GFP reporter under control of the REG3 $\gamma$ promoter. Furthermore, we investigated the hypothesis that absence of REG3 $\gamma$ would result in higher susceptibility to infection with Gram-positive L. monocytogenes than to infection with Gramnegative $S$. enteritidis, due to its proposed bactericidal activity towards Gram-positive bacteria.

Initial phenotypic histological analysis revealed no morphological differences between REG3 $\gamma^{-1-}$ and wt mice, corroborating another REG3 $\gamma^{-1-}$ mouse study. ${ }^{14}$ However, we observed a significant increase in the relative amounts of IL-22 transcript and MPO protein levels in tissue samples from the ileum of REG3 $\gamma^{-/-}$mice relative to wt mice, suggesting elevated innate responses and chemotaxis of neutrophils.

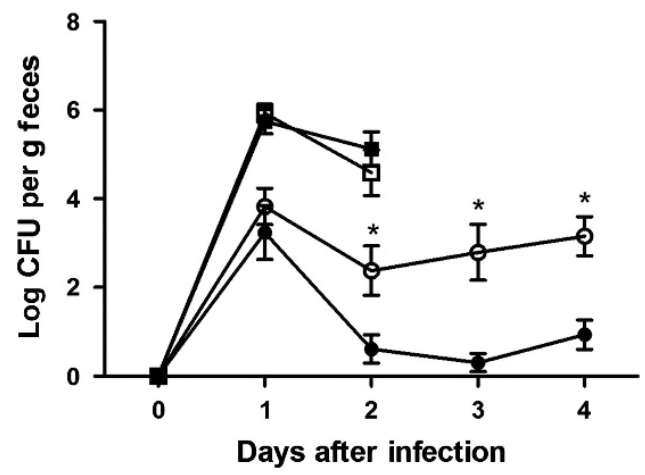

Figure 5 Pathogens excreted (CFU) in the feces at different time points after infection. One $S$. enteritidis infected wt mouse was not included in this figure because it did not produce feces. L. monocytogenes CFU are shown as squares and $S$. enteritidis CFU as circles. Open symbols represent CFU of REG3 $\gamma^{-1-}$ mice and filled symbols CFU of wt mice. Error bars indicate s.e.m.; ${ }^{*}=P<0.05$, comparing $S$. enteritidis infected REG3 $\gamma^{-1-}$ vs wt mice; CFU, colony forming units; wt, wild-type.

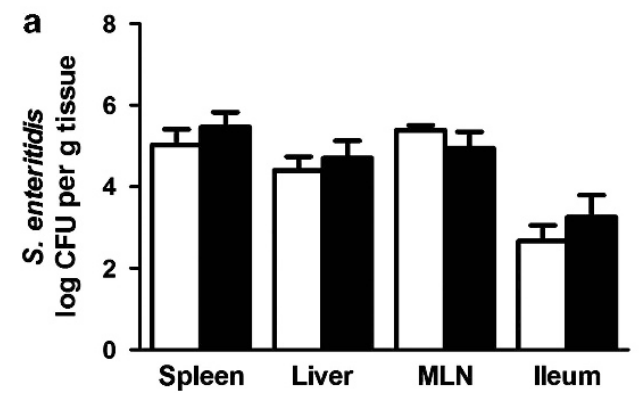

Moreover, significantly higher amounts of the chemokines MCP-1 and M-CSF-1, both of which are involved in monocyte and neutrophil recruitment, activation and differentiation, were found in serum. Interestingly, pro-inflammatory cytokines, such as IL-1 $\beta$, IL-12p70, TNF- $\alpha$, and IFN- $\gamma$, were not different between REG3 $\gamma^{-/-}$and wt mice, or were below detection limit. Therefore, it appears that REG3 $\gamma^{-/-}$mice exhibit an increased innate immune cell activity in the mucosa that successfully avoids systemic inflammation, which is a new finding compared with the previous work published about the in vivo lack of REG3 $\gamma$. $^{14}$

As MUC2, the major intestinal mucin, is highly glycosylated and REG3 $\gamma$, a C-type lectin, is able to bind glycans, we hypothesized that loss of REG3 $\gamma$ might make the mucus more accessible for bacteria and would allow increased contact between microbiota and epithelia. Indeed, sections of ileal tissue from REG3 $\gamma^{-1-}$ mice, but not wt mice, consistently showed the presence of small numbers of bacteria at the epithelium, in agreement with the study of Vaishnava et al. ${ }^{14}$ However, an important difference in our study is the absence of segmented filamentous bacteria in our mice which appeared to be substantially increased in the study of Vaishnava et al. ${ }^{14}$ Given their unique effects on Th17 cell differentiation and IgA production in mice, they may account for much of the previous observations. In the colon, increased co-localization of bacteria

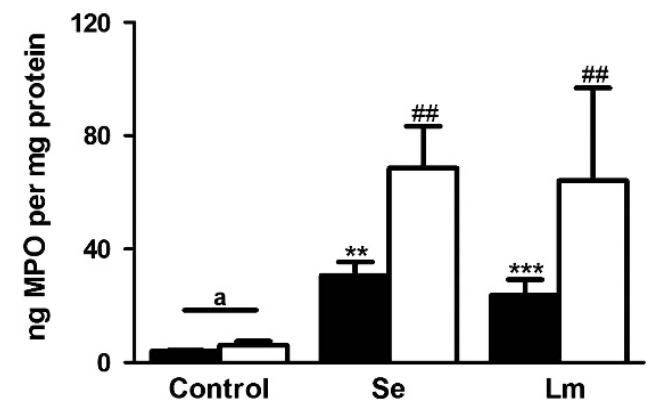

Figure 7 Amount of MPO in ileal protein extracts of wt (black bars) and REG3 $\gamma^{-1-}$ mice (white bars) measured by ELISA. Significant difference between wt infected and uninfected mice, ${ }^{* \star} P=<0.01,{ }^{* * *} P=<0.005$; Significant difference between infected and uninfected REG3 $\gamma^{-1-}$ mice, ${ }^{\# \#} P=<0.01$. MPO was also significantly different between uninfected REG3 $\gamma^{-1-}$ and wt mice $\left({ }^{\mathrm{a}} P=<0.05\right)$. Se, S. enteritidis;

Lm, L. monocytogenes; MPO, myeloperoxidase; wt, wild-type.

Figure 6 Pathogens (CFU, colony forming units) in ileum and organs of mice infected with S. enteritidis (a) and L. monocytogenes (b). White bars indicate log CFU per g of tissue from REG3 $\gamma^{-1-}$ mice and black bars indicate log CFU per g of tissue from wt mice. Error bars indicate s.e.m. No significant differences between REG $3 \gamma^{-1-}$ and wt mice were observed. MLN, mesenteric lymph node. wt, wild-type. 
a
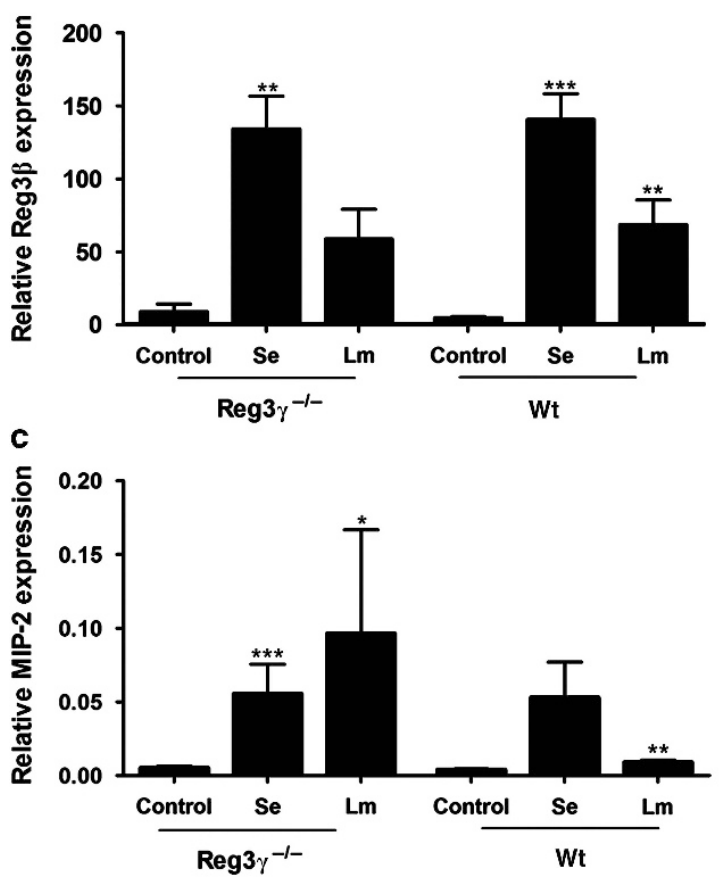

b

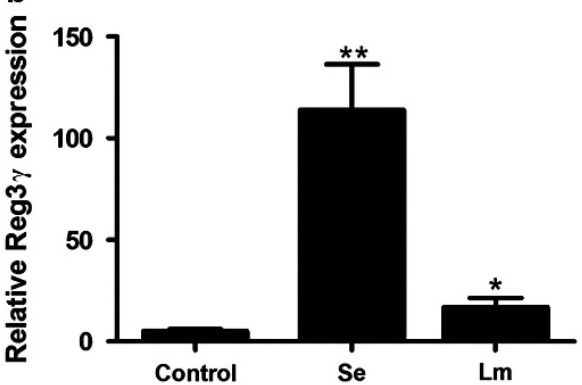

d

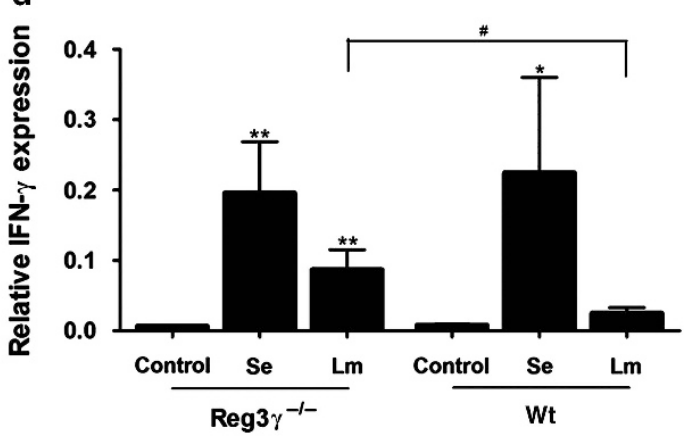

Figure 8 Relative mRNA expression of immune-related genes in uninfected and infected mice determined by qPCR. REG3 $\beta$ (a); REG3 $\gamma$ (b); MIP-2 (c) and IFN- $\gamma(\mathbf{d})$. Error bars indicate s.e.m. Significant difference between infected and uninfected REG $3 \gamma^{-1-}$ or wt mice, ${ }^{\star} P=<0.05$; ${ }^{\star \star}$ significance $P=<0.01^{* * *} P=<0.005$. Se, S. enteritidis; Lm, L. monocytogenes; wt, wild-type.

and epithelial surfaces was not observed, presumably due to the thicker mucus layer ${ }^{15}$ and the lower levels of REG3 $\gamma$ expression in the large intestine. ${ }^{4} \mathrm{We}$ next investigated the distribution and location of the mucus in wt and REG $3 \gamma^{-/-}$mice. Strikingly, mucus visualization revealed an intensely stained band of mucus consistently located above the villi and surrounding the ileal content in wt mice; this band was absent in REG3 $\gamma^{-/-}$ mice. This was not reported by Vaishnava et al. ${ }^{14}$ as the mucus was not visualized in that study. The changes in mucus were not due to lower expression of MUC2 mRNA in the ileum of REG3 $\gamma^{-1-}$ mice, suggesting that REG3 $\gamma$ may directly affect the structure or the distribution of mucus in the ileum. As REG3 $\gamma$ was shown to be present throughout the mucus layer of wt mice, its absence may provide an explanation for the altered distribution of mucus in these REG3 $\gamma^{-1-}$ mice. Alternatively, REG3 $\gamma$ itself may self-assemble into large multimeric complexes as previously described for PAP and other REG family members ${ }^{13}$ and stabilize the mucin structure by crosslinking the mucins in the mucus network. We have seen binding of REG3 $\gamma$ to colonic mucus (data not shown) supporting the idea of a direct effect of REG3 $\gamma$ on the stability or penetrability of the mucus layer in the ileum.

As binding of REG3 $\gamma$ to bacteria might be a mechanism to trap bacteria in the mucus, we investigated whether recombinant REG3 $\gamma$ could bind both pathogens in vitro. Indeed REG3 $\gamma$ could bind to both pathogens and binding was enhanced by proteolytic cleavage of the $\mathrm{N}$-terminal propeptide (data not shown, methods in supplement). Recombinant REG3 $\gamma$ is not bactericidal for $S$. enteritidis (not shown) as shown for other Gram-negative bacteria by Cash et al. ${ }^{5}$ Therefore the significant decrease in the fecal counts of Gram-negative S. enteritidis in wt mice compared with REG3 $\gamma^{-1-}$ mice may be due to increased entrapment of $S$. enteritidis in the mucus by REG3 $\gamma$, and subsequent killing by antimicrobial proteins including REG3 $\beta$, which is highly increased in expression after S. enteritidis infection. An alternative explanation is that increased luminal counts of $S$. enteritidis are due to changes in the composition or distribution of the epithelial-associated microbiota. REG3 $\gamma$ was not bactericidal against the L. monocytogenes strain, which was previously reported to be killed by REG3 $\gamma$ and HIP/PAP, ${ }^{5}$ but inconsistencies in the bactericidal effect of REG3 $\gamma$ and HIP/ PAP on Gram-positive bacteria have been described by others. ${ }^{12,13}$ This discrepancy may be due to the use of different bacteria, possible interference of bacterial aggregation during viability assays or conformational differences in the refolded proteins leading to differential proteolytic susceptibility or activity. Lack of REG3 $\gamma$ and concomitant decreased bacterial entrapment could thereby explain the increased mucosal inflammatory responses observed following infection of REG3 $\gamma^{-1-}$ mice with either pathogen.

Infection of REG3 $\gamma^{-1-}$ mice with either of the pathogens did not result in increased translocation to the spleen, liver or MLNs compared with wt infected mice. This result was fully reproduced using a "murinized" L. monocytogenes, engineered to increase infectivity in murine models. ${ }^{16}$ The lack of higher colonization levels of $L$. monocytogenes to the ileum of REG3 $\gamma^{-/-}$mice was unexpected as Vaishnava et al. ${ }^{14}$ reported increased mucosal-associated Gram-positive bacteria in REG3 $\gamma^{-1-}$ mice. However, at the bacterial group level, this was shown to be due to Eubacterium rectale and segmented 
filamentous bacteria, of which the latter is not present in our mice. Although there was no increased translocation of pathogens in the organs of REG3 $\gamma^{-1-}$ mice, we did measure increased amounts of MPO in ileal tissue of infected REG3 $\gamma^{-1-}$ mice compared with wt infected mice. MPO is produced mostly by neutrophil granulocytes, which are attracted to sites of infection suggesting that increased numbers of both pathogens colonized the mucosa of REG3 $\gamma^{-1-}$ mice compared with wt mice. Taken together, these results suggest that the heightened mucosal inflammatory response observed in infected REG3 $\gamma^{-1-}$ mice was sufficient to inhibit spread of the pathogens to the bloodstream and organs. In agreement with this, loss of REG3 $\gamma$ did not affect systemic inflammatory responses as no significant differences between wt and REG3 $\gamma^{-1-}$ in serum SAA2 levels were measured. In line with this notion, it was observed that several genes in the infection response network were differentially expressed in REG3 $\gamma^{-1-}$ compared with wt mice. For example, in L. monocytogenes infected mice, If $n-\gamma$, Il-1 $\beta$, Icam 1, and Cxcl9 were expressed at relatively higher levels in REG3 $\gamma^{-1-}$ mice than in wt mice.

Overall these results suggest that the mucosal inflammatory response, involving monocyte and neutrophil recruitment and activity to pathogen infection, was increased in REG3 $\gamma^{-1-}$ mice. Reduced trapping of the bacteria in the mucus or increased movement of bacteria through the mucus might explain the increased mucosal inflammatory responses to both pathogens in REG $3 \gamma^{-1-}$ mice. Our results showing that presence of REG3 $\gamma$ throughout the mucus altered the mucus distribution in the ileum of REG3 $\gamma^{-1-}$ mice, and REG3 $\gamma$ binding to both pathogens support this hypothesis.

By generating a new REG3 $\gamma^{-1-}$ mouse model, we were able to demonstrate that REG3 $\gamma$ deficiency leads to altered mucus distribution, increased bacterial-epithelial contact, and significant increases in the expression of several innate immunity genes in the ileum. During infection, REG3 $\gamma$ deficiency had no effect on translocation of pathogenic L. monocytogenes or $S$. enteritidis to the organs, but resulted in a heightened innate response in the mucosa. The increased amount of MPO measured in the mucosa of REG3 $\gamma^{-1-}$ mice suggests the presence of higher numbers of polymorphonuclear granulocytes which are important in immunity to both L. monocytogenes and S. enteritidis. In addition, our results suggest that REG3 $\gamma$ may have protective effects that go beyond direct bactericidal activity, including effects on mucus properties and trapping of bacteria in the mucus network.

\section{METHODS}

Animals. REG3 $\gamma^{-1-}$ mice were generated using a Cre-Lox procedure and the genotype was verified by PCR (see Supplementary Materials and Methods and Supplementary Table S1 for primer sequences). Specified pathogen free REG3 $\gamma^{-1-}$ and wt mice on a $\mathrm{C} 57 \mathrm{Bl} / 6$ background were reared on a semi-synthetic standard AIN-93G $\operatorname{diet}^{19}$ (Research Diet Services, Wijk bij Duurstede, the Netherlands) and provided with sterilized drinking water ad libitum, unless stated otherwise. The animal welfare committee of Wageningen University (Wageningen, the Netherlands) approved the experimental protocol.
Collection of biological samples for phenotyping. Mice (7-9 weeks old) were anaesthetized by isoflurane, blood was collected after orbital removal using BD Microtainer tubes (SST tubes, Becton Dickinson (BD), Franklin Lakes, NJ) and mice were killed by cervical dislocation. Serum was analyzed by Myriad-RBM (Rules Based Medicine; Austin, TX) using the RodentMAP v2.0 platform. Different parts (approximately $1 \mathrm{~cm}$ ) of the ileum and colon were either fixed in Carnoy's fixative or in $4 \%(\mathrm{w} / \mathrm{v})$ paraformaldehyde (PFA) and embedded in paraffin. PFA-fixed tissue sections were stained according to Crossmon, Carnoy's fixed tissue sections were stained with PAS/Alcian Blue (PAS/AB), anti-Muc2 antibody ${ }^{15}$ or fluorescence in situ hybridization (FISH) to detect bacteria using the universal bacterial probe EUB338 ${ }^{18}$ (for histological protocols, see supplement). Unfixed tissue was snap frozen and stored at $-80{ }^{\circ} \mathrm{C}$ prior to RNA isolation. Mesenteric lymph nodes (MLNs) were collected, the cells cultured and stimulated ex vivo for cytokine analysis (Supplementary Methods).

Infection studies. Mice (7-9 weeks old) used in the infection study were housed individually from one week before the start of the infection and fed a modified diet, to mimic a Western-style human diet, by including $200 \mathrm{~g} \mathrm{~kg}^{-1}$ fat and reducing the calcium to $30 \mathrm{mmol} \mathrm{kg}-1$. The amount of calcium in a standard rodent diet is typically very high $\left(124.75 \mathrm{mmol} \mathrm{kg}^{-1} \mathrm{feed}^{17}\right)$ but we lowered this because it has been shown to be protective against infection in the intestine of rats ${ }^{19,20}$ and humans. ${ }^{21}$ This "humanized" diet was fed to the mice from 7 weeks of age. Moreover, this diet has been also used in a previous infection study using REG3 $\beta^{-1-}$ mice. ${ }^{22}$

After adaptation, mice were orally infected by gavage with $0.2 \mathrm{ml}$ saline containing $10^{8}$ colony forming units (CFU) of Salmonella enterica serovar enteritidis (S. enteritidis, $n=12$ for REG3 $\gamma^{-1-}$ and wt) or L. monocytogenes (EGD-e, $n=8$ for REG3 $\gamma^{-1-}$ and wt). The bacterial strains were passaged in mice and enumerated on selective medium as previously described. ${ }^{22}$ Two groups of mice were sham treated $\left(0.2 \mathrm{ml}\right.$ saline, REG3 $\gamma^{-1-}$ and wt; both $\left.n=6\right)$. An additional infection study was performed with non-passaged murinized L. monocytogenes ${ }^{16}$ (see supplement). Body weight was measured every two days before infection and daily after infection. Fresh feces were collected every day after infection.

Collection of biological samples and bacterial quantification of infected mice. Mice were sacrificed 2 (L. monocytogenes) or 4 days (S. enteritidis) after infection, the time points at which the number of translocated bacteria were highest in the organs. ${ }^{22}$ Non-infected control mice were sacrificed 3 days after oral sham treatment. Blood was collected as above and after cervical dislocation, the distal onethird of the small intestine was excised. For determining CFU of pathogens (shown as $\log _{10}$ CFU per gram wet weight of tissue), approximately $1 \mathrm{~cm}$ from the middle of the ileum was cut open longitudinally, briefly flushed in saline, homogenized in sterile saline, and plated. Other parts of the ileum were treated in the same way and frozen immediately in liquid nitrogen for protein and/or RNA isolation (see supplementary for protocols and Supplementary Table S2 for primer sequences for qPCR). Furthermore, MLNs, spleen, and liver were removed and after homogenization in sterile saline, directly used for pathogen quantification.

Myeloperoxidase quantification in ileal mucosa and serum amyloid A2 detection in serum. To determine the ileal inflammation status, MPO was measured in ileal protein extracts using the manufacturer's MPO ELISA protocol (Hycult Biotechnology, Uden, the Netherlands). Serum Amyloid A (SAA) 2, was measured to investigate systemic inflammation according to the manufacturer's protocol (Life Diagnostics, West Chester, PA).

Microarray and statistical analysis. Total RNA was extracted from the ileum as described above, pooled (three animals per group, randomly selected), and hybridized to the Mouse Gene 1.1 ST array 
(Affymetrix, Santa Clara, CA). The labeling and hybridization methods, quality control procedures on RNA and data sets generated are described in Supplementary Methods.

Biological interpretation of expression data sets. Biological interaction networks among regulated genes activated in response to infection were identified using Ingenuity Pathways Analysis (IPA) (Ingenuity Systems, Redwood City, CA) using all differentially regulated genes $(P$ value $<0.01$ and intensity $\geq 20)$ as input. The output was used to prioritize differentially regulated pathways of interest for bacterial infections, to identify cascades of upstream transcriptional regulators that could explain the observed gene expression changes, and to reconstruct protein-protein networks that could be used to overlay gene expression data. For details, see Supplementary Methods.

Statistics. All statistical tests were performed using GraphPad Prism 5.0 software (GraphPad, San Diego, CA). Data shown are the means and the standard errors of the means (s.e.m.). Data were tested for normality with the D'Agostino-Pearson normality test. Statistical analysis of normally distributed data was performed with the two-tailed Student's $t$-test. Data that did not show normal distribution were analyzed with the non-parametric Mann-Whitney $U$ test. The excretion of pathogens in feces was measured at multiple time points, and thus analyzed by a repeated-measures two-way analysis of variance (ANOVA) (mixed model). Differences were considered statistically significant when $P<0.05$.

SUPPLEMENTARY MATERIAL is linked to the online version of the paper at http://www.nature.com/mi

\section{ACKNOWLEDGMENTS}

We want to express our gratitude to Colin Hill (University College Cork, Ireland) for providing the murinized $L$. monocytogenes strain EGD-e. Furthermore we want to thank Gunnar Hansson (University of Gotherburg, Sweden) for providing the anti-MUC2 antibody. We are grateful to the biotechnicians of the small animal facility of the Wageningen University (Wageningen, the Netherlands), Nico Taverne and Anja Taverne-Thiele (Host-Microbe interactomics group, Wageningen University) for their excellent technical assistance. Bruno Sovran (Host-Microbe interactomics group, Wageningen University) is thanked for fruitful discussions and for providing protocols. Finally Jenny Janssen (Division of Human Nutrition, Wageningen University) is thanked for her technical assistance with the micro-arrays. This research was funded by TI Food and Nutrition, a publicprivate partnership on pre-competitive research in food and nutrition. The funders had no role in study design, data collection and analysis, decision to publish, or preparation of the manuscript.

\section{DISCLOSURE}

The authors declared no conflicts of interest.

c) 2014 Society for Mucosal Immunology

\section{REFERENCES}

1. Corthesy, B. Role of secretory immunoglobulin $A$ and secretory component in the protection of mucosal surfaces. Future Microbiol. 5, 817-829 (2010).

2. Ouellette, A.J. Paneth cells and innate mucosal immunity. Curr. Opin. Gastroenterol. 26, 547-553 (2010).

3. Frigerio, J.M. et al. The pancreatitis associated protein III (PAP III), a new member of the PAP gene family. Biochim. Biophys. Acta 1216, 329-331 (1993).
4. Burger-van Paassen, N. et al. Mucin Muc2 deficiency and weaning influences the expression of the innate defense genes Reg3 $\beta$, Reg3 $\gamma$ and Angiogenin-4. PLoS One 7, e38798 (2012).

5. Cash, H.L. et al. Symbiotic bacteria direct expression of an intestinal bactericidal lectin. Science 313, 1126-1130 (2006).

6. Narushima, Y. et al. Structure, chromosomal localization and expression of mouse genes encoding type III Reg, Reglll[alpha], Reglll[beta], Reglll[gamma]. Gene 185, 159-168 (1997).

7. Christa, L. et al. HIP/PAP is an adhesive protein expressed in hepatocarcinoma, normal Paneth, and pancreatic cells. Am. J. Physiol. 271 (6 Pt 1), G993-1002 (1996).

8. Ogawa, H. etal. Increased expression of HIP/PAP and regenerating gene III in human inflammatory bowel disease and a murine bacterial reconstitution model. Inflamm. Bowel Dis. 9, 162-170 (2003).

9. van Ampting, M.T. et al. Ileal mucosal and fecal pancreatitis associated protein levels reflect severity of Salmonella infection in rats. Dig. Dis. Sci. 54, 2588-2597 (2009).

10. Dessein, R. et al. Toll-like receptor 2 is critical for induction of Reg3 beta expression and intestinal clearance of Yersinia pseudotuberculosis. Gut 58, 771-776 (2009).

11. Gironella, M. et al. Anti-inflammatory effects of pancreatitis associated protein in inflammatory bowel disease. Gut 54, 1244-1253 (2005).

12. Iovanna, J, Frigerio, J.M., Dusetti, N, Ramare, F, Raibaud, P \& Dagorn, J.C. Lithostathine, an inhibitor of $\mathrm{CaCO} 3$ crystal growth in pancreatic juice, induces bacterial aggregation. Pancreas 8, 597-601 (1993).

13. Medveczky, P, Szmola, R \& Sahin-Toth, M. Proteolytic activation of human pancreatitis-associated protein is required for peptidoglycan binding and bacterial aggregation. Biochem. J. 420, 335-343 (2009).

14. Vaishnava, S. et al. The antibacterial lectin Reglllgamma promotes the spatial segregation of microbiota and host in the intestine. Science 334 , 255-258 (2011).

15. Johansson, M.E., Phillipson, M, Petersson, J, Velcich, A, Holm, L \& Hansson, G.C. The inner of the two Muc2 mucin-dependent mucus layers in colon is devoid of bacteria. Proc. Natl. Acad. Sci. USA 105, 15064-15069 (2008).

16. Monk, I.R., Casey, P.G., Hill, C \& Gahan, C.G. Directed evolution and targeted mutagenesis to murinize Listeria monocytogenes internalin A for enhanced infectivity in the murine oral infection model. BMC Microbiol. 10, 318 (2010).

17. Reeves, P.G., Nielsen, F.H. \& Fahey, G.C. Jr AIN-93 purified diets for laboratory rodents: final report of the American Institute of Nutrition ad hoc writing committee on the reformulation of the AIN-76A rodent diet. J. Nutr. 123, 1939-1951 (1993).

18. Johansson, M.E. \& Hansson, G.C. Preservation of mucus in histological sections, immunostaining of mucins in fixed tissue, and localization of bacteria with FISH. Methods Mol. Biol. 842, 229235 (2012)

19. Bovee-Oudenhoven, I.M., Termont, D.S., Heidt, P.J. \& Van der Meer, R. Increasing the intestinal resistance of rats to the invasive pathogen Salmonella enteritidis: additive effects of dietary lactulose and calcium. Gut 40, 497-504 (1997).

20. Bovee-Oudenhoven, I.M., Termont, D.S., Weerkamp, A.H., FaassenPeters, M.A. \& Van der Meer, R. Dietary calcium inhibits the intestinal colonization and translocation of Salmonella in rats. Gastroenterology 113, 550-557 (1997).

21. Bovee-Oudenhoven, I.M., Lettink-Wissink, M.L., Van Doesburg, W., Witteman, B.J. \& Van Der Meer, R. Diarrhea caused by enterotoxigenic Escherichia coli infection of humans is inhibited by dietary calcium. Gastroenterology 125, 469-476 (2003).

22. van Ampting, M.T. et al. Intestinally secreted C-type lectin Reg3b attenuates salmonellosis but not listeriosis in mice. Infect. Immun. 80, 1115-1120 (2012) 